\title{
Structure of the Functionally Important Extracellular Loop C of Human Aquaporin 1 Obtained by Solid-State NMR under Nearly Physiological Conditions
}

Dylan Archer Dingwell, Leonid S. Brown*, Vladimir Ladizhansky*

Department of Physics and Biophysics Interdepartmental Group, University of Guelph, 50 Stone Road E, Guelph, ON, N1G 2W1, Canada

Address correspondence to Leonid S. Brown, lebrown@uoguelph.ca; Vlad Ladizhansky, vladizha@uoguelph.ca.

Supporting Information 


\section{S1. Sample Preparation}

Transformant cell stocks stored at $-80^{\circ} \mathrm{C}$ were replated onto yeast peptone dextrose (YPD, Table S1) plates with high concentration of zeocin (Cedarlane, $1000 \mu \mathrm{g} / \mathrm{mL}$ ) and incubated at $30{ }^{\circ} \mathrm{C}$. After $4-7$ days of growth, a single well-isolated colony was transferred to 50 $\mathrm{mL}$ isotope-labeled ${ }^{13} \mathrm{C},{ }^{15} \mathrm{~N}$ buffered minimal dextrose (BMD, $\mathrm{pH}$ 6.0, Table S1) media in a 250 $\mathrm{mL}$ sterile shake-flask and grown at $30^{\circ} \mathrm{C}$ for $18-24$ hours. Cultures were then transferred to $1 \mathrm{~L}$ sterile shake-flasks containing additional $200 \mathrm{~mL}$ BMD media and grown at $29^{\circ} \mathrm{C}$ and $275 \mathrm{rpm}$ for another 18-24 hours. Cells were collected by centrifugation at $1500 \times \mathrm{g}$ rcf for 10 minutes at 4 ${ }^{\circ} \mathrm{C}$ in sterile containers and resuspended in $1 \mathrm{~L}$ isotope-labeled ${ }^{13} \mathrm{C},{ }^{15} \mathrm{~N}$ buffered minimal methanol (BMM, pH 6.0, Table S1) media in a sterile $2.8 \mathrm{~L}$ shake-flask. Cells were grown for additional 24 hours at $28^{\circ} \mathrm{C}, 240 \mathrm{rpm}$, and then collected by centrifugation at $1500 \times \mathrm{g} \mathrm{rcf}$ for 10 minutes at $4{ }^{\circ} \mathrm{C}$ and washed twice with MilliQ water. Cell pellets were stored at $-20{ }^{\circ} \mathrm{C}$ for later use.

Frozen cell pellets were resuspended in one pellet volume of cell resuspension buffer (CRB, $\mathrm{pH}$ 7.6, Table S1) with addition of equivalent volume of acid-washed glass beads (Fisher, 420-600 $\mu \mathrm{m}$ diameter). Cells were broken by vortex mixing for 1 minute followed by a 1 minute interval in which the suspension was rested on ice; this pattern was repeated 4 times, following which cell debris was separated via low-speed centrifugation at $700 \times \mathrm{g}$ rcf for 5 minutes at $4{ }^{\circ} \mathrm{C}$ and cell lysate was collected. Eight rounds of vortexing were completed with collection of additional lysate for complete breakage. Combined supernatants containing cell lysate from all rounds of breakage were centrifuged at $150,000 \times \mathrm{g} \operatorname{rcf}$ for 1 hour at $4{ }^{\circ} \mathrm{C}$, and the pellet containing membrane fractions was stored at $-20^{\circ} \mathrm{C}$ for later use. 
Solubilization of hAQP1 was achieved by resuspending the frozen membrane fraction pellet in $20 \mathrm{~mL}$ solubilization buffer $(\mathrm{pH} 8.0$, Table $\mathrm{S} 1)$ and stirring gently at $4{ }^{\circ} \mathrm{C}$ for 2 hours. Unsolubilized material was removed by centrifugation at $150,000 \times \mathrm{g}$ rcf for 1 hour, and $2-5 \mathrm{~mL}$ $\mathrm{Ni}^{2+}$-NTA resin (Qiagen) was then added to the supernatant containing solubilized hAQP1. This solution was incubated overnight with gentle stirring to allow complete resin binding, then placed in a column and the buffer was flown through. The resin was washed with $500 \mathrm{~mL}$ washing buffer ( $\mathrm{pH}$ 8.0, Table S1) and hAQP1 was eluted using 20-40 mL elution buffers (pH 8.0, Table S1) with increasing concentrations of imidazole. Purified hAQP1 in elution buffer was stored at $4{ }^{\circ} \mathrm{C}$ for no more than 7 days, during which the elution volume was concentrated to 5 $\mathrm{mL}$ with concurrent removal of imidazole (Amicon centrifugal concentrator, $10 \mathrm{kDa}$ cut-off, Fisher) and buffer exchange to reconstitution buffer (pH 7.5, Table S1).

For lipid reconstitution, purified hAQP1 in imidazole-free reconstitution buffer was incubated overnight with gentle stirring at $4{ }^{\circ} \mathrm{C}$, with prepared lipid stock (egg PC:brain PS, 9:1 $\mathrm{w} / \mathrm{w}$, Avanti lipids) at an approximate protein:lipid weight ratio of 2:1. Following incubation with lipids, detergent removal was accomplished by dialysis. The sample was placed into a dialysis bag (12-14 kDa cutoff, Spectra/Por, VWR) fixed at the top of a container with 200-500 $\mathrm{mL}$ dialysis buffer ( $\mathrm{pH} 7.5$, Table $\mathrm{S} 1$ ) and $8 \mathrm{~g}$ of Bio-beads SM-2 (Biorad), and the dialysis container was stirred gently at $4{ }^{\circ} \mathrm{C}$. Dialysis continued for 7-10 days with changing of dialysis buffer and addition of 1-4 g Bio-beads every 24 to 48 hours, until no detergent was present. The reconstituted proteoliposome suspension was collected from the dialysis bag and collected by centrifugation at $150,000 \times \mathrm{g}$ rcf for 1 hour at $4{ }^{\circ} \mathrm{C}$, and then washed with NMR buffer $(\mathrm{pH} 7.0$, Table S1) by centrifugation at $300,000 \times \mathrm{g}$ rcf for 30 minutes at $4{ }^{\circ} \mathrm{C}$. Finally, proteoliposomes in NMR buffer were centrifuged at $900,000 \times \mathrm{g}$ rcf for 16 hours at $4{ }^{\circ} \mathrm{C}$, and the resulting pellet was 
ready for SSNMR rotor packing. Unpacked sample at this stage was stored at $-20{ }^{\circ} \mathrm{C}$ for later use.

\section{Table S1. Buffers for hAQP1 Sample Preparation}

\begin{tabular}{|l|r|}
\hline Yeast Peptone Dextrose (YPD), $\mathbf{p H = 6 . 5}$ & $1 \%$ \\
\hline yeast extract & $2 \%$ \\
\hline peptone & $2 \%$ \\
\hline dextrose & \\
\hline
\end{tabular}

\begin{tabular}{|l|r|}
\hline Buffered Minimal Dextrose (BMD), $\mathbf{p H}=\mathbf{6 . 0}$ & $1 \%$ \\
\hline ammonium sulfate & $100 \mathrm{mM}$ \\
\hline potassium phosphate & $0.34 \%$ \\
\hline yeast nitrogen base & $0.00004 \%$ \\
\hline biotin & $0.5 \%$ \\
\hline dextrose &
\end{tabular}

\begin{tabular}{|l|r|}
\hline Buffered Minimal Methanol (BMM), $\mathbf{p H}=\mathbf{6 . 0}$ & $1 \%$ \\
\hline ammonium sulfate & $100 \mathrm{mM}$ \\
\hline potassium phosphate & $0.34 \%$ \\
\hline yeast nitrogen base & $0.00004 \%$ \\
\hline biotin & $0.5 \%$ \\
\hline methanol &
\end{tabular}

\begin{tabular}{|l|r|}
\hline Cell Resuspension Buffer (CRB), $\mathbf{p H = 7 . 6}$ & $20 \mathrm{mM}$ \\
\hline Tris-HCl & $100 \mathrm{mM}$ \\
\hline sodium chloride $\mathrm{NaCl}$ & $0.5 \mathrm{mM}$ \\
\hline EDTA & $5 \%(\mathrm{w} / \mathrm{v})$ \\
\hline glycerol &
\end{tabular}

\begin{tabular}{|l|r|}
\hline Solubilization Buffer, $\mathbf{p H}=\mathbf{8 . 0}$ & $20 \mathrm{mM}$ \\
\hline Tris-HCl & $100 \mathrm{mM}$ \\
\hline sodium chloride $\mathrm{NaCl}$ & $20 \%$ \\
\hline glycerol & $10 \mathrm{mM}$ \\
\hline imidazole & $1 \mathrm{X}$ \\
\hline protease inhibitor cocktail (for general use, Sigma) & $5 \%$ \\
\hline$n$-octyl- $\beta$-D-glucopyranoside & \\
\hline
\end{tabular}

\begin{tabular}{|l|r|}
\hline Washing Buffer, $\mathbf{p H}=\mathbf{8 . 0}$ & $20 \mathrm{mM}$ \\
\hline Tris-HCl & $100 \mathrm{mM}$ \\
\hline sodium chloride $\mathrm{NaCl}$ & $20 \%$ \\
\hline glycerol &
\end{tabular}




\begin{tabular}{|l|r|}
\hline imidazole & $30 \mathrm{mM}$ \\
\hline protease inhibitor cocktail (for general use, Sigma) & $1 \mathrm{X}$ \\
\hline$n$-octyl- $\beta$-D-glucopyranoside & $1 \%$ \\
\hline
\end{tabular}

\begin{tabular}{|l|r|}
\hline Elution Buffer, $\mathbf{p H}=\mathbf{8 . 0}$ \\
\hline Tris-HCl & $20 \mathrm{mM}$ \\
\hline sodium chloride $\mathrm{NaCl}$ & $100 \mathrm{mM}$ \\
\hline glycerol & $20 \%$ \\
\hline imidazole & $150-500 \mathrm{mM}$ \\
\hline protease inhibitor cocktail (for general use, Sigma) & $1 \mathrm{X}$ \\
\hline$n$-octyl- $\beta$-D-glucopyranoside & $1 \%$ \\
\hline
\end{tabular}

\begin{tabular}{|l|r|}
\hline Reconstitution Buffer, $\mathbf{p H}=\mathbf{7 . 5}$ & $50 \mathrm{mM}$ \\
\hline potassium phosphate & $100 \mathrm{mM}$ \\
\hline sodium chloride & \\
\hline
\end{tabular}

\begin{tabular}{|l|r|}
\hline Dialysis Buffer, $\mathbf{p H}=\mathbf{7 . 5}$ & $50 \mathrm{mM}$ \\
\hline potassium phosphate & $300 \mathrm{mM}$ \\
\hline sodium chloride & \\
\hline
\end{tabular}

\begin{tabular}{|l|r|}
\hline NMR Buffer, $\mathbf{p H}=\mathbf{7 . 0}$ & $25 \mathrm{mM}$ \\
\hline Tris-HCl & $10 \mathrm{M}$ \\
\hline sodium chloride & \\
\hline
\end{tabular}

\section{S2. NMR Experiments}

3D NCOCX HBR ${ }^{2}$ experiments were performed using pulse sequence in Figure $\mathrm{S} 1 .{ }^{1} \mathrm{H} /{ }^{15} \mathrm{~N}$ cross polarization $(\mathrm{CP})^{1}$ of $2 \mathrm{~ms}$ duration was implemented with an ${ }^{15} \mathrm{~N}$ field strength of $35 \mathrm{kHz}$ and with the proton field ramped $10 \%$ around $n=2$ Hartmann-Hahn matching condition ${ }^{2} .{ }^{15} \mathrm{~N} /{ }^{13} \mathrm{C}$, band selective $\mathrm{CP}^{3}$ was performed with a 6 ms contact pulse with a spinlock fields on ${ }^{15} \mathrm{~N}$ at the field strength corresponding to $2.5 \mathrm{x}$ spinning frequency and with the carbon field intensity ramped linearly $(10 \%)$ around $3.5 \mathrm{x}$ spinning frequency. SPINAL- $64^{4}$ decoupling was used during direct and indirect chemical shift evolution. Typical recycle delays were $1.5 \mathrm{~s}$. Additional details on the $\mathrm{HBR}^{2}$ mixing can be found in Table S2. 


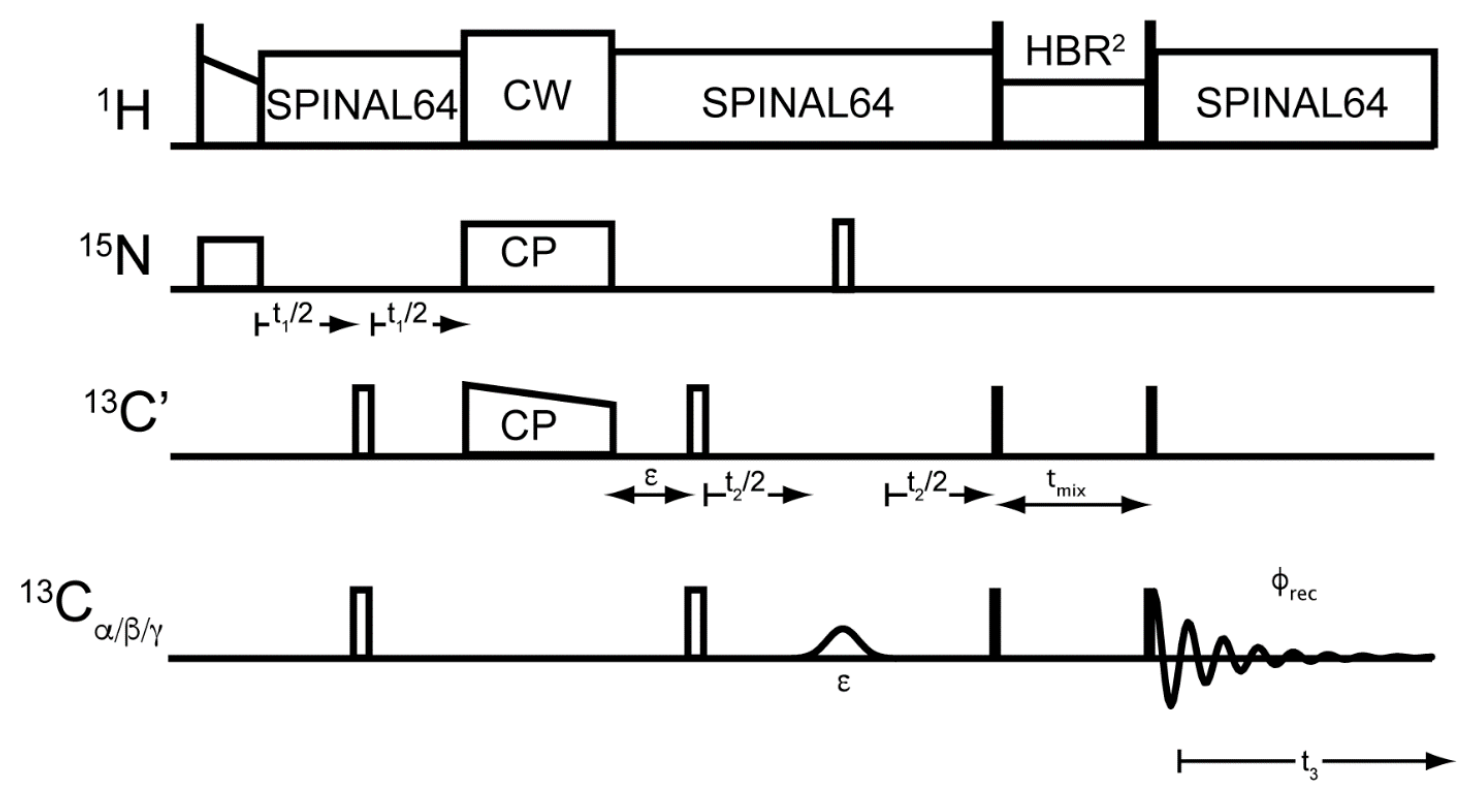

Figure S1. SSNMR pulse sequence used for 3D NCOCX $\mathrm{HBR}^{2}$ correlation experiments. N-CO polarization transfers use SPECIFIC CP; CO-CX HBR ${ }^{2}$ mixing was implemented with 70-100 ms CW irradiation of $38-50 \mathrm{kHz}$ applied to protons (Table S2). An off-resonance selective Gaussian cascade refocusing pulse of $350 \mu \mathrm{sec}$ duration was used to refocus $\mathrm{C} \alpha-\mathrm{CO}$ homonuclear J-couplings. 2D HBR ${ }^{2}$ experiments used this pulse sequence with $\mathrm{t}_{2}=0$.

Table S2. Summary of 3D HBR ${ }^{2}$ NCOCX experiments.

Table S2A

\begin{tabular}{|l|l|}
\hline Proton frequency $[\mathrm{MHz}]$ & 800.23 \\
\hline Spinning frequency $[\mathrm{kHz}]$ & 15.0 \\
\hline Acquisition length $\left(\mathrm{t}_{3} / \mathrm{t}_{2} / \mathrm{t}_{1}\right)[\mathrm{ms}]$ & $20.9 / 9.5 / 11.2$ \\
\hline $\mathrm{HBR}^{2}$ mixing time $[\mathrm{ms}]$ & 100 \\
\hline $\mathrm{CW}$ decoupling power during $\mathrm{HBR}^{2}$ mixing $[\mathrm{kHz}]$ & 38 \\
\hline
\end{tabular}

Table S2B

\begin{tabular}{|l|l|}
\hline Proton frequency $[\mathrm{MHz}]$ & 800.23 \\
\hline Spinning frequency $[\mathrm{kHz}]$ & 14.6 \\
\hline Acquisition length $\left(\mathrm{t}_{3} / \mathrm{t}_{2} / \mathrm{t}_{1}\right)[\mathrm{ms}]$ & $20.9 / 9.5 / 11.2$ \\
\hline $\mathrm{HBR}^{2}$ mixing time $[\mathrm{ms}]$ & 100 \\
\hline $\mathrm{CW}$ decoupling power during $\mathrm{HBR}^{2}$ mixing $[\mathrm{kHz}]$ & 39 \\
\hline
\end{tabular}

Table S2C

\begin{tabular}{|l|l|}
\hline Proton frequency $[\mathrm{MHz}]$ & 800.23 \\
\hline Spinning frequency $[\mathrm{kHz}]$ & 14.3 \\
\hline Acquisition length $\left(\mathrm{t}_{3} / \mathrm{t}_{2} / \mathrm{t}_{1}\right)[\mathrm{ms}]$ & $20.9 / 9.5 / 11.2$ \\
\hline $\mathrm{HBR}^{2}$ mixing time $[\mathrm{ms}]$ & 100 \\
\hline $\mathrm{CW}$ decoupling power during $\mathrm{HBR}^{2}$ mixing $[\mathrm{kHz}]$ & 39 \\
\hline
\end{tabular}


Table S2D

\begin{tabular}{|l|l|}
\hline Proton frequency $[\mathrm{MHz}]$ & 800.23 \\
\hline Spinning frequency $[\mathrm{kHz}]$ & 15.6 \\
\hline Acquisition length $\left(\mathrm{t}_{3} / \mathrm{t}_{2} / \mathrm{t}_{1}\right)[\mathrm{ms}]$ & $20.9 / 9.3 / 11.1$ \\
\hline $\mathrm{HBR}^{2}$ mixing time $[\mathrm{ms}]$ & 70 \\
\hline $\mathrm{CW}$ decoupling power during $\mathrm{HBR}^{2}$ mixing $[\mathrm{kHz}]$ & 41 \\
\hline
\end{tabular}

Table S2E

\begin{tabular}{|l|l|}
\hline Proton frequency $[\mathrm{MHz}]$ & 800.23 \\
\hline Spinning frequency $[\mathrm{kHz}]$ & 14.0 \\
\hline Acquisition length $\left(\mathrm{t}_{3} / \mathrm{t}_{2} / \mathrm{t}_{1}\right)[\mathrm{ms}]$ & $20.9 / 9.3 / 11.1$ \\
\hline $\mathrm{HBR}^{2}$ mixing time $[\mathrm{ms}]$ & 70 \\
\hline $\mathrm{CW}$ decoupling power during $\mathrm{HBR}^{2}$ mixing $[\mathrm{kHz}]$ & 48 \\
\hline
\end{tabular}

Table S2F

\begin{tabular}{|l|l|}
\hline Proton frequency $[\mathrm{MHz}]$ & 800.23 \\
\hline Spinning frequency $[\mathrm{kHz}]$ & 15.9 \\
\hline Acquisition length $\left(\mathrm{t}_{3} / \mathrm{t}_{2} / \mathrm{t}_{1}\right)[\mathrm{ms}]$ & $20.9 / 9.3 / 11.1$ \\
\hline $\mathrm{HBR}^{2}$ mixing time $[\mathrm{ms}]$ & 70 \\
\hline $\mathrm{CW}$ decoupling power during $\mathrm{HBR}^{2}$ mixing $[\mathrm{kHz}]$ & 43 \\
\hline
\end{tabular}

Table S2G

\begin{tabular}{|l|l|}
\hline Proton frequency $[\mathrm{MHz}]$ & 800.23 \\
\hline Spinning frequency $[\mathrm{kHz}]$ & 14.8 \\
\hline Acquisition length $\left(\mathrm{t}_{3} / \mathrm{t}_{2} / \mathrm{t}_{1}\right)[\mathrm{ms}]$ & $20.9 / 9.3 / 11.1$ \\
\hline $\mathrm{HBR}^{2}$ mixing time $[\mathrm{ms}]$ & 70 \\
\hline $\mathrm{CW}$ decoupling power during $\mathrm{HBR}^{2}$ mixing $[\mathrm{kHz}]$ & 40 \\
\hline
\end{tabular}

Table S2H

\begin{tabular}{|l|l|}
\hline Proton frequency $[\mathrm{MHz}]$ & 600.13 \\
\hline Spinning frequency $[\mathrm{kHz}]$ & 11.5 \\
\hline Acquisition length $\left(\mathrm{t}_{3} / \mathrm{t}_{2} / \mathrm{t}_{1}\right)[\mathrm{ms}]$ & $23.9 / 7.0 / 10.3$ \\
\hline $\mathrm{HBR}^{2}$ mixing time $[\mathrm{ms}]$ & 70 \\
\hline $\mathrm{CW}$ decoupling power during $\mathrm{HBR}^{2}$ mixing $[\mathrm{kHz}]$ & 50 \\
\hline
\end{tabular}

\section{Table S2I}

\begin{tabular}{|l|l|}
\hline Proton frequency $[\mathrm{MHz}]$ & 600.13 \\
\hline Spinning frequency $[\mathrm{kHz}]$ & 11.1 \\
\hline Acquisition length $\left(\mathrm{t}_{3} / \mathrm{t}_{2} / \mathrm{t}_{1}\right)[\mathrm{ms}]$ & $23.9 / 7.0 / 10.3$ \\
\hline $\mathrm{HBR}^{2}$ mixing time $[\mathrm{ms}]$ & 70 \\
\hline $\mathrm{CW}$ decoupling power during $\mathrm{HBR}^{2}$ mixing $[\mathrm{kHz}]$ & 50 \\
\hline
\end{tabular}



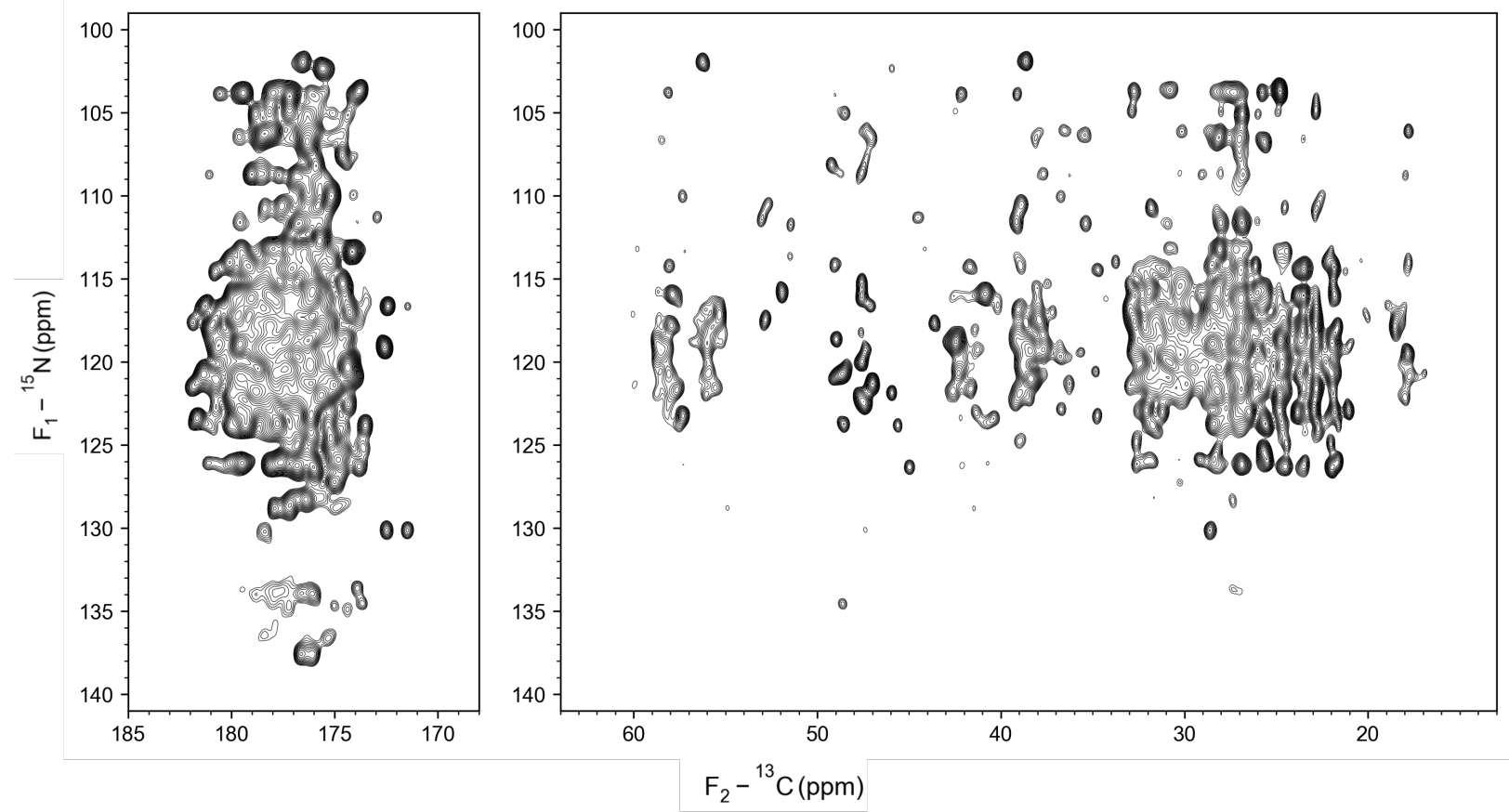

Figure S2. 2D HBR ${ }^{2}$ spectrum of hAQP1 collected on the $800 \mathrm{MHz}$ spectrometer at a spinning frequency of $15 \mathrm{KHz}$. Most peaks in the carbonyl region (185-170 ppm, carbon dimension) and belonging to sidechain carbons (40-20 ppm, carbon dimension) are overlapped, preventing identification of structurally constraining cross-peak correlations. Cross peaks in the ${ }^{13} \mathrm{C} \alpha$ region reflect residual recoupling of the $\mathrm{CO}-\mathrm{C} \alpha$ interactions.

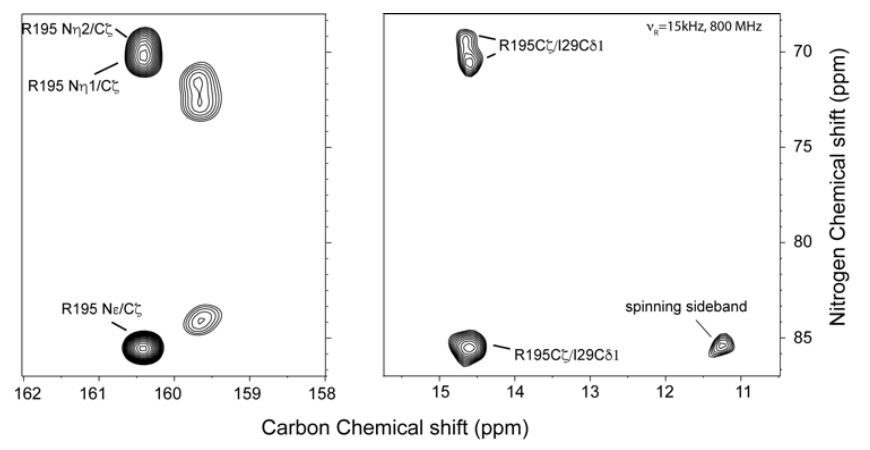

Figure S3. R195C $\zeta-I 29 \mathrm{C} \delta 1$ correlations obtained in the 2D HBR ${ }^{2}$ spectrum of hAQP1 collected on the $800 \mathrm{MHz}$ spectrometer at a spinning frequency of $15 \mathrm{KHz}$. 

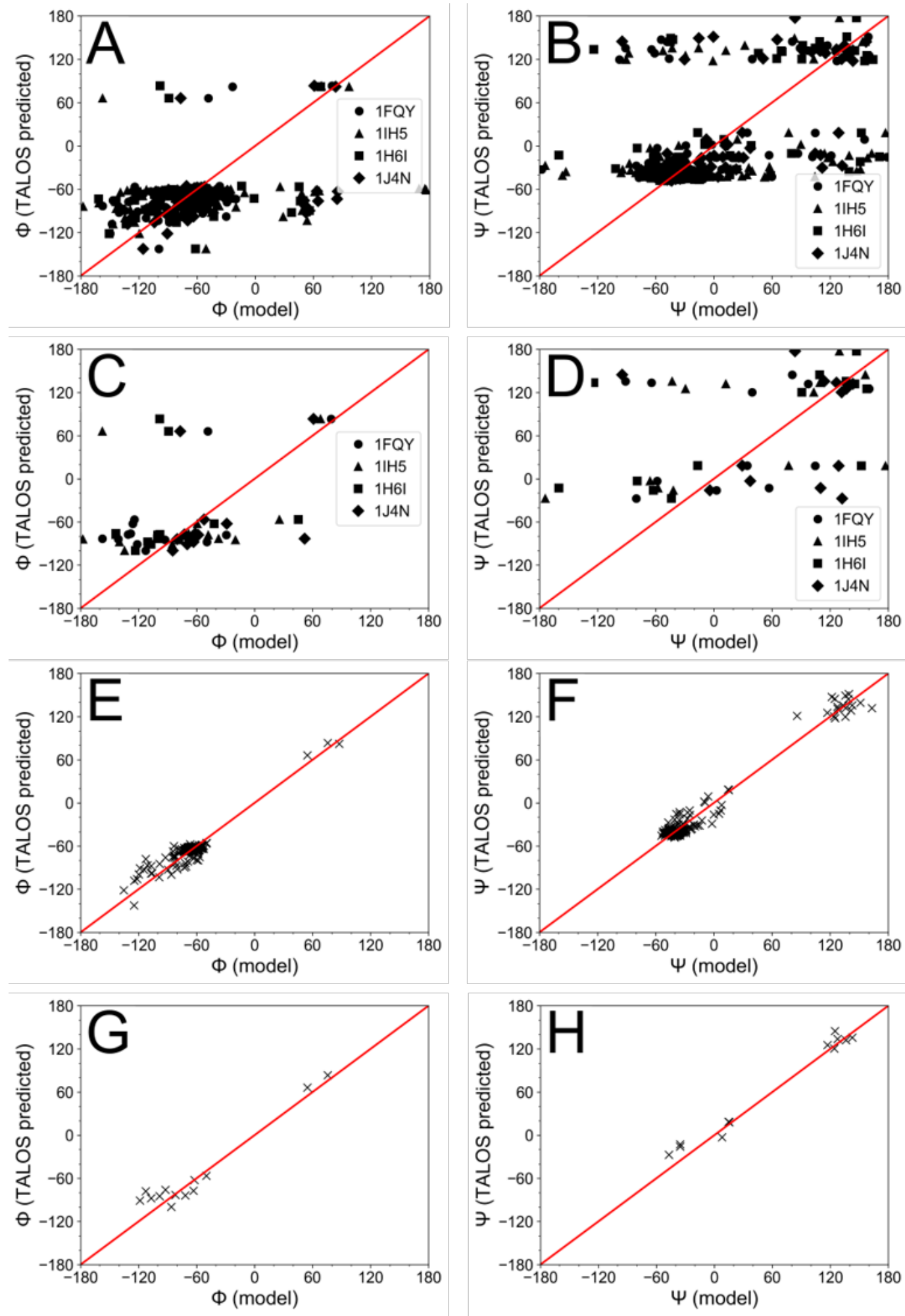

Figure S4. Observed dihedral angles compared with TALOS-predicted dihedral angles for 1FQY, 1IH5, 1H6I structures of hAQP1 as well homology model based on bAQP1 structure $1 \mathrm{~J} 4 \mathrm{~N}$ (A-D), and for final refined model (E-H). Comparison is shown for all residues for phi $(\mathrm{A}, \mathrm{E})$ and psi $(\mathrm{B}, \mathrm{F})$ angles, as well as loop $\mathrm{C}$ residues only $\phi(\mathrm{C}, \mathrm{G})$ and $\psi(\mathrm{D}, \mathrm{H})$. 

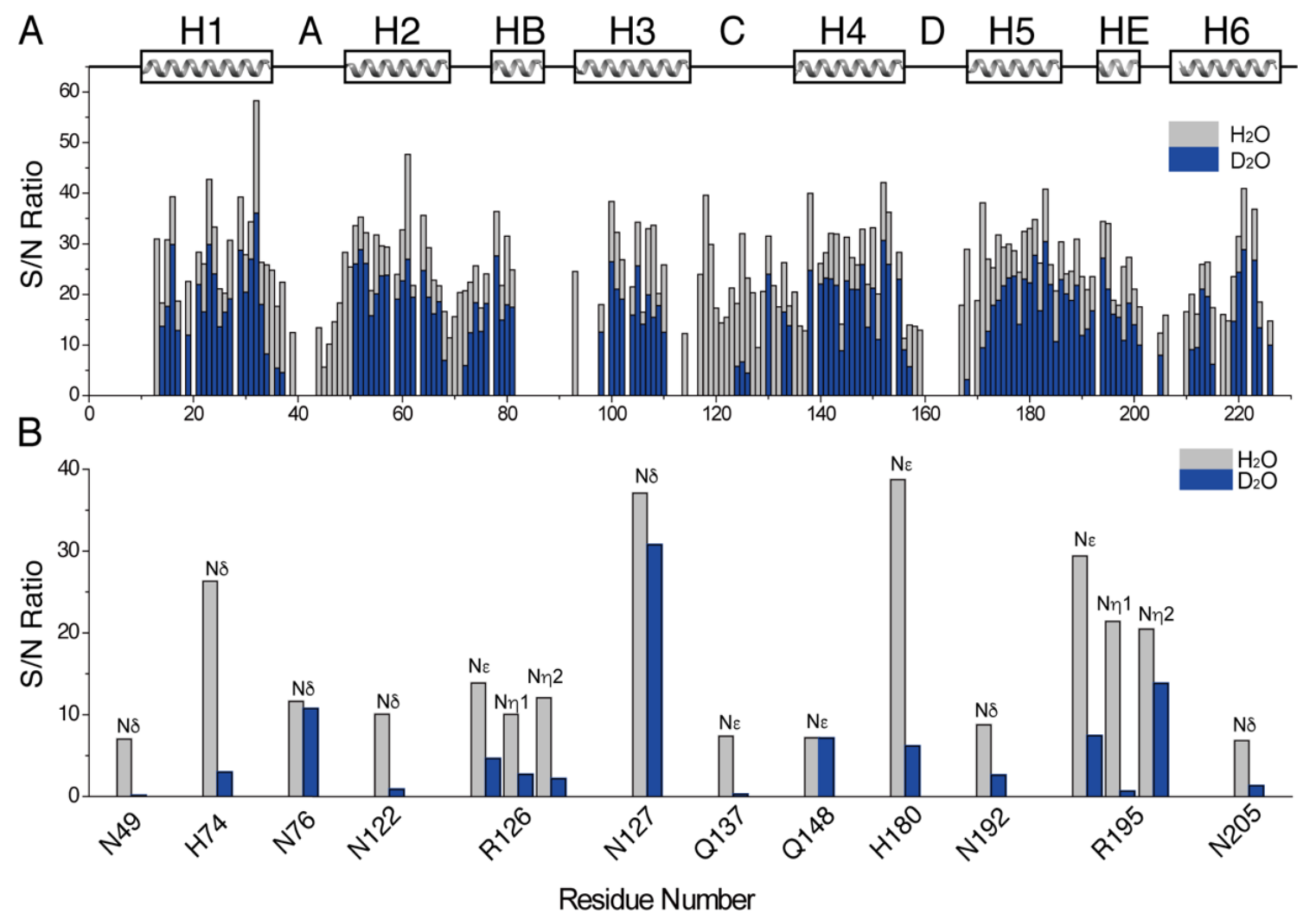

Figure S5. Hydrogen-deuterium exchange experiments. (A) Backbone amide cross peak intensities from hAQP1 incubated in $\mathrm{H}_{2} \mathrm{O}$ (grey) and $\mathrm{D}_{2} \mathrm{O}$ (blue) based buffers. (B) Nitrogenbearing side chain signal intensities detected in $\mathrm{H}_{2} \mathrm{O}$ (grey) and $\mathrm{D}_{2} \mathrm{O}$ (blue). $\mathrm{H} / \mathrm{D}$ exchange results in signal attenuation below ca. $20-25 \%$. Reproduced with permission from ref. 5 . Copyright 2016 American Chemical Society. 

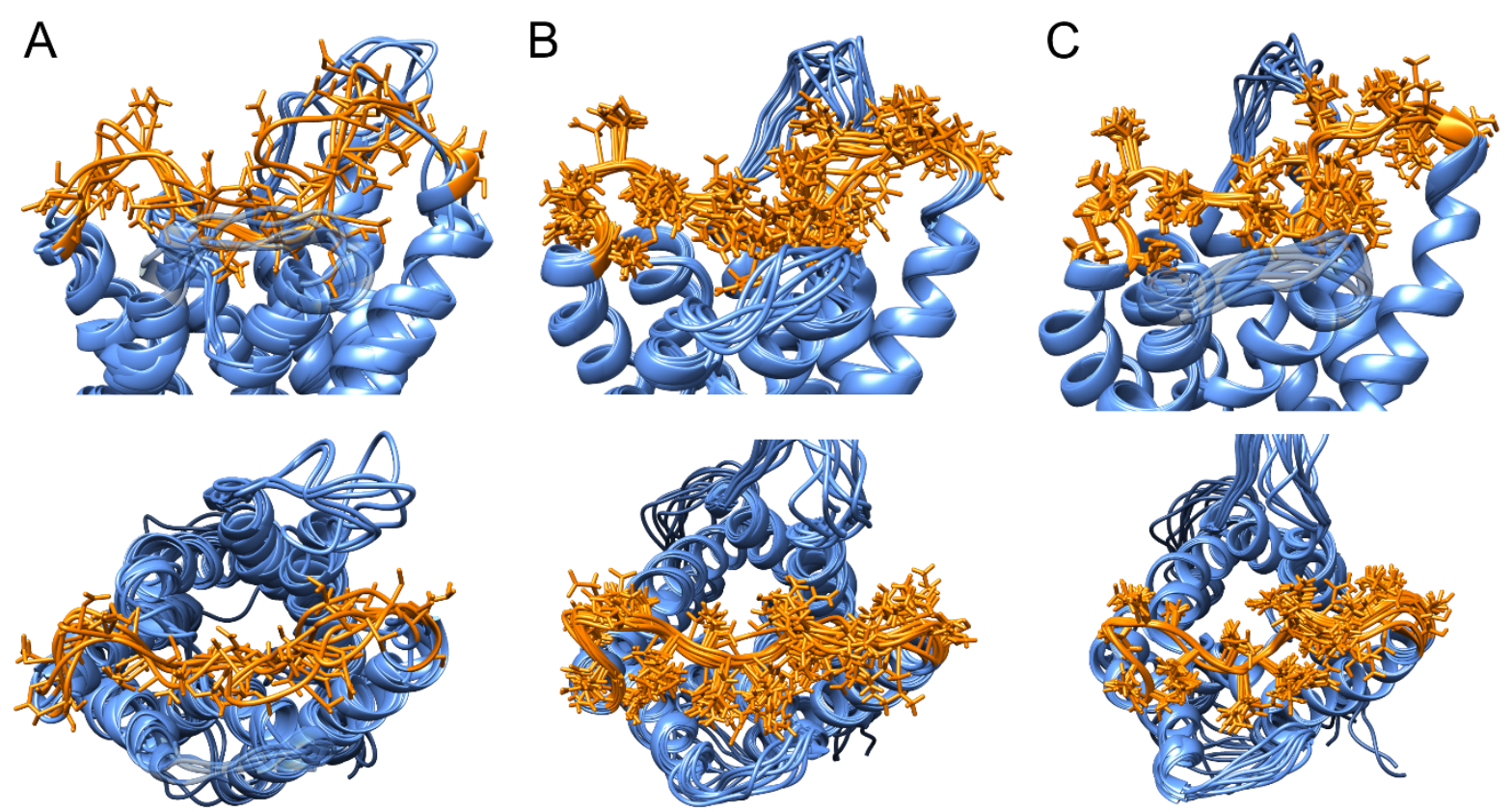

Figure S6. Convergence of the loop $\mathrm{C}$ sidechain conformations (highlighted orange) through stages of iterative structure calculation. (A) Template models including all existing PDB structures and homology model based on bAQP1. (B) Ensemble of 10 lowest-energy models after three rounds of refinement calculation. (C) Final ensemble of 10 lowest-energy models after seven rounds of refinement calculation. Side view is shown on top, with the extracellular side oriented upward. View from the extracellular side is shown in the bottom row.

Table S3. List of distance restraints used to calculate the final refined model of hAQP1. Initially identified long-range unambiguous restraints are shown in red font. Restraints involving loop $\mathrm{C}$ are indicated in bold.

\begin{tabular}{|l|l|l|l|}
\hline CO Residue \# & CO Spin & CX Residue \# & CX Spin \\
\hline 15 & C & 17 & CB \\
\hline 15 & C & 19 & CA \\
\hline 15 & C & 13 & CA \\
\hline 17 & C & 101 & CB \\
\hline 17 & C & 19 & CB \\
\hline 18 & C & 20 & CB \\
\hline 23 & C & 27 & CD1 \\
\hline 24 & C & 27 & CD1 \\
\hline 24 & C & 28 & CA \\
\hline 27 & C & 29 & CB \\
\hline 27 & C & 29 & CG1 \\
\hline 27 & C & 29 & CA \\
\hline 29 & C & 33 & CG \\
& & & \\
\hline
\end{tabular}




\begin{tabular}{|c|c|c|c|}
\hline 29 & $\mathrm{C}$ & 31 & CA \\
\hline 31 & $\mathrm{C}$ & 35 & CB \\
\hline 33 & $\mathrm{C}$ & 37 & CA \\
\hline 34 & C & 36 & CG \\
\hline 36 & C & 34 & $\mathrm{CA}$ \\
\hline 36 & $\mathrm{C}$ & 39 & CB \\
\hline 37 & C & 34 & CA \\
\hline 47 & C & 51 & CG \\
\hline 48 & $\mathrm{C}$ & 51 & CG \\
\hline 48 & $\mathrm{C}$ & 51 & CE \\
\hline 49 & C & 53 & $\mathrm{CB}$ \\
\hline 49 & C & 181 & CG \\
\hline 49 & CG & 184 & $\mathrm{CG} 2$ \\
\hline 50 & $\mathrm{C}$ & 181 & CD1 \\
\hline 51 & $\mathrm{C}$ & 55 & $\mathrm{CA}$ \\
\hline 56 & C & 176 & CG1 \\
\hline 58 & C & 61 & CA \\
\hline 58 & $\mathrm{C}$ & 62 & CG2 \\
\hline 58 & C & 60 & $\mathrm{CB}$ \\
\hline 58 & C & 61 & CB \\
\hline 59 & C & 61 & CB \\
\hline 59 & $\mathrm{C}$ & 63 & CB \\
\hline 59 & C & 62 & CB \\
\hline 59 & C & 62 & CG2 \\
\hline 61 & C & 63 & CB \\
\hline 61 & $\mathrm{C}$ & 63 & CA \\
\hline 62 & C & 65 & CA \\
\hline 62 & $\mathrm{C}$ & 64 & CB \\
\hline 64 & C & 67 & $\mathrm{CG} 2$ \\
\hline 64 & $\mathrm{C}$ & 62 & CA \\
\hline 65 & $\mathrm{C}$ & 62 & $\mathrm{CA}$ \\
\hline 65 & C & 67 & CG2 \\
\hline 71 & $\mathrm{C}$ & 64 & CB \\
\hline 71 & $\mathrm{C}$ & 64 & CA \\
\hline 72 & C & 74 & CB \\
\hline 73 & C & 75 & CG \\
\hline 74 & $\mathrm{C}$ & 20 & CB \\
\hline 75 & $\mathrm{C}$ & 21 & CG2 \\
\hline 75 & C & 21 & CA \\
\hline 75 & C & 101 & $\mathrm{CG}$ \\
\hline 75 & $\mathrm{C}$ & 20 & CB \\
\hline 75 & $\mathrm{C}$ & 73 & CB \\
\hline 75 & C & 21 & $\mathrm{CB}$ \\
\hline 78 & C & 149 & CD2 \\
\hline 78 & C & 150 & CG2 \\
\hline
\end{tabular}




\begin{tabular}{|c|c|c|c|}
\hline 95 & $\mathrm{C}$ & 98 & CD1 \\
\hline 95 & $\mathrm{C}$ & 98 & CG2 \\
\hline 96 & $\mathrm{C}$ & 94 & CA \\
\hline 103 & $\mathrm{C}$ & 18 & CA \\
\hline 103 & C & 18 & CB \\
\hline 103 & $\mathrm{C}$ & 106 & CB \\
\hline 103 & C & 106 & CG1 \\
\hline 103 & $\mathrm{C}$ & 101 & CA \\
\hline 103 & $\mathrm{C}$ & 105 & CA \\
\hline 103 & $\mathrm{C}$ & 107 & CG2 \\
\hline 108 & C & 26 & CB \\
\hline 108 & $\mathrm{C}$ & 22 & CG2 \\
\hline 109 & C & 112 & CB \\
\hline 113 & $\mathrm{C}$ & 115 & $\mathrm{CB}$ \\
\hline 114 & C & 116 & CA \\
\hline 120 & $\mathbf{C}$ & 122 & CB \\
\hline 123 & C & 125 & CA \\
\hline 124 & $\mathrm{C}$ & 29 & CG2 \\
\hline 124 & C & 126 & CB \\
\hline 124 & $\mathbf{C}$ & 32 & CB \\
\hline 127 & C & 129 & CD2 \\
\hline 130 & C & 132 & CA \\
\hline 134 & C & 129 & CG \\
\hline 134 & C & 187 & CA \\
\hline 134 & C & 137 & CB \\
\hline 134 & C & 137 & CG \\
\hline 135 & C & 137 & CB \\
\hline 135 & $\mathbf{C}$ & 138 & CA \\
\hline 135 & C & 133 & CG1 \\
\hline 136 & $\mathbf{C}$ & 133 & CG1 \\
\hline 137 & C & 139 & CB \\
\hline 137 & C & 133 & CB \\
\hline 137 & $\mathrm{C}$ & 141 & $\mathrm{CB}$ \\
\hline 137 & $\mathbf{C}$ & 133 & CG2 \\
\hline 137 & C & 141 & CD1 \\
\hline 139 & C & 142 & CB \\
\hline 139 & C & 143 & $\mathrm{CB}$ \\
\hline 140 & $\mathrm{C}$ & 143 & CD1 \\
\hline 141 & C & 138 & CA \\
\hline 142 & $\mathrm{C}$ & 146 & CA \\
\hline 142 & $\mathrm{C}$ & 145 & CA \\
\hline 142 & $\mathrm{C}$ & 146 & CB \\
\hline 143 & C & 147 & $\mathrm{CB}$ \\
\hline 144 & C & 148 & $\mathrm{CB}$ \\
\hline 145 & C & 191 & CG2 \\
\hline
\end{tabular}




\begin{tabular}{|c|c|c|c|}
\hline 145 & C & 191 & CD1 \\
\hline 146 & $\mathrm{C}$ & 148 & CA \\
\hline 146 & $\mathrm{C}$ & 148 & CG \\
\hline 147 & $\mathrm{C}$ & 150 & CB \\
\hline 152 & $\mathrm{C}$ & 172 & CB \\
\hline 155 & $\mathrm{C}$ & 157 & CA \\
\hline 167 & $\mathrm{C}$ & 169 & CG \\
\hline 167 & $\mathrm{C}$ & 170 & CD1 \\
\hline 168 & $\mathrm{C}$ & 172 & CB \\
\hline 168 & $\mathrm{C}$ & 172 & CG1 \\
\hline 168 & C & 171 & CB \\
\hline 171 & $\mathrm{C}$ & 174 & CA \\
\hline 172 & $\mathrm{C}$ & 176 & CG2 \\
\hline 175 & $\mathrm{C}$ & 179 & CA \\
\hline 175 & $\mathrm{C}$ & 173 & CA \\
\hline 175 & $\mathrm{C}$ & 148 & CG \\
\hline 176 & $\mathrm{C}$ & 149 & CD1 \\
\hline 176 & $\mathrm{C}$ & 178 & CB \\
\hline 177 & $\mathrm{C}$ & 180 & CB \\
\hline 177 & $\mathrm{C}$ & 181 & CB \\
\hline 178 & $\mathrm{C}$ & 181 & CG \\
\hline 178 & $\mathrm{C}$ & 181 & CD1 \\
\hline 178 & $\mathrm{C}$ & 182 & $\mathrm{CD} 2$ \\
\hline 178 & $\mathrm{C}$ & 180 & CB \\
\hline 178 & $\mathrm{C}$ & 182 & CG \\
\hline 179 & $\mathrm{C}$ & 181 & CA \\
\hline 179 & $\mathrm{C}$ & 191 & CD1 \\
\hline 180 & $\mathrm{C}$ & 184 & CB \\
\hline 180 & $\mathrm{C}$ & 184 & CG1 \\
\hline 180 & $\mathrm{C}$ & 184 & CD \\
\hline 182 & $\mathrm{C}$ & 186 & CB \\
\hline 183 & $\mathrm{C}$ & 187 & CG2 \\
\hline 185 & $\mathrm{C}$ & 130 & CB \\
\hline 185 & $\mathrm{C}$ & 187 & CG2 \\
\hline 185 & $\mathrm{C}$ & 188 & CA \\
\hline 186 & $\mathbf{C}$ & 130 & CB \\
\hline 187 & $\mathbf{C}$ & 130 & CB \\
\hline 188 & $\mathrm{C}$ & 190 & CA \\
\hline 188 & $\mathrm{C}$ & 184 & CG2 \\
\hline 189 & $\mathrm{C}$ & 184 & CG1 \\
\hline 189 & $\mathrm{C}$ & 184 & CB \\
\hline 189 & $\mathrm{C}$ & 196 & CB \\
\hline 189 & $\mathrm{C}$ & 187 & CG2 \\
\hline 189 & $\mathrm{C}$ & 187 & CB \\
\hline 190 & C & 183 & CB \\
\hline
\end{tabular}




\begin{tabular}{|c|c|c|c|}
\hline 190 & C & 195 & $\mathrm{CB}$ \\
\hline 191 & $\mathrm{C}$ & 146 & $\mathrm{CG} 2$ \\
\hline 191 & C & \begin{tabular}{|l|}
78 \\
\end{tabular} & CB \\
\hline 191 & $\mathrm{C}$ & 216 & $\mathrm{CG}$ \\
\hline 192 & $\mathrm{C}$ & 190 & CA \\
\hline 192 & C & 78 & CB \\
\hline 193 & $\mathrm{C}$ & \begin{tabular}{|l|l}
77 \\
\end{tabular} & CG \\
\hline 193 & C & 196 & CB \\
\hline 195 & $\mathrm{CZ}$ & 29 & CD1 \\
\hline 195 & $\mathrm{CZ}$ & 125 & CA \\
\hline 195 & C & 190 & CA \\
\hline 197 & $\mathrm{C}$ & 106 & CG1 \\
\hline 200 & $\mathrm{C}$ & 197 & CA \\
\hline 210 & $\mathrm{C}$ & 212 & $\mathrm{CB}$ \\
\hline 211 & $\mathrm{C}$ & 214 & CG1 \\
\hline 212 & $\mathrm{C}$ & 193 & CB \\
\hline 212 & $\mathrm{C}$ & 216 & CG \\
\hline 212 & $\mathrm{C}$ & 216 & CD \\
\hline 213 & $\mathrm{C}$ & 216 & $\mathrm{CG}$ \\
\hline 214 & $\mathrm{C}$ & 216 & CG \\
\hline 214 & $\mathrm{C}$ & 218 & CB \\
\hline 214 & C & 217 & CA \\
\hline 218 & C & 222 & CD1 \\
\hline 218 & $\mathrm{C}$ & 222 & CA \\
\hline 219 & $\mathrm{C}$ & 222 & CB \\
\hline 219 & C & 147 & CB \\
\hline 219 & $\mathrm{C}$ & 222 & CD1 \\
\hline 219 & $\mathrm{C}$ & $\begin{array}{ll}147 \\
\end{array}$ & CD1 \\
\hline 219 & C & 143 & CG2 \\
\hline 219 & $\mathrm{C}$ & 223 & CB \\
\hline 220 & $\mathrm{C}$ & 222 & $\mathrm{CG}$ \\
\hline 220 & $\mathrm{C}$ & 150 & CG2 \\
\hline 222 & $\mathrm{C}$ & 224 & CB \\
\hline 222 & $\mathrm{C}$ & 226 & CB \\
\hline
\end{tabular}


Table S4. Refinement statistics for hAQP1 structure calculation

\section{NMR distance and dihedral restraints}

Distance restraints

Total restraints

2608

Total restraints for loop C

197

Intra-residue

1368

Intra-residue for loop C

109

Inter-residue

Sequential $(|i-j|=1)$

Sequential for loop C

1058

Short-range $(|i-j|=2)$

67

Short-range for loop C

50

Medium-range $(2<|i-j|<5)$

7

Medium-range for loop $\mathrm{C}$

Long-range $(|i-j|>4)$

81

6

Long-range for loop $\mathrm{C}$

Dihedral angle restraints

$\psi$

$\phi$

Cis-bonds

177

Dihedral restraints for loop C

1 (Proline-38)

$\psi$

13

13

\section{Restraints statistics}

Violations

Distance restraints $(\AA)$

Max. distance restraint violation

$0.069 \pm 0.001$

Deviation from idealized geometry

Bond lengths $(\AA)$

0.35

Bond angles $\left({ }^{\circ}\right)$

$0.007 \pm 0.000$

$0.809 \pm 0.008$

\section{Structural precision}

Root-mean-square-deviation $(\AA)$

Heavy atoms

$2.88 \pm 0.23$

Heavy atoms for loop C

$1.49 \pm 0.46$

Backbone

$1.87 \pm 0.25$

Backbone for loop C

$0.85 \pm 0.44$

\section{Ramachandran Plot statistics ${ }^{\mathrm{a}}$}

Residues in most favored region (\%)

Residues in additional allowed region (\%) $\quad 12.5$

Residues in generously allowed region (\%) $\quad 0.5$

Residues in disallowed region (\%) 1.6

${ }^{a}$ Evaluated by PROCHECK. ${ }^{6,7}$ 


\section{References}

(1) Pines, A.; Gibby, M. G.; Waugh, J. S. Proton-enhanced NMR of dilute spins in solids. $J$. Chem. Phys. 1973, 59, 569-590.

(2) Hartmann, S. R.; Hahn, E. L. Nuclear Double Resonance in the Rotating Frame. Phys. Rev. 1962, 128, 2042-2053.

(3) Baldus, M.; Petkova, A. T.; Herzfeld, J.; Griffin, R. G. Cross Polarization in the Tilted Frame: Assignment and Spectral Simplification in Heteronuclear Spin Systems. Mol. Phys. 1998, 95, 1197-1207.

(4) Fung, B. M.; Khitrin, A. K.; Ermolaev, K. An Improved Broadband Decoupling Sequence for Liquid Crystals and Solids. J. Magn. Reson. 2000, 142, 97-101.

(5) Wang, S.; Ing, C.; Emami, S.; Jiang, Y.; Liang, H.; Pomès, R.; Brown, L. S.; Ladizhansky, V. Structure and Dynamics of Extracellular Loops in Human Aquaporin-1 from Solid-State NMR and Molecular Dynamics. J. Phys. Chem. B 2016, 120 (37), 9887-9902.

(6) Laskowski, R. A.; MacArthur, M. W.; Moss, D. S.; Thornton, J. M. PROCHECK: A Program to Check the Stereochemical Quality of Protein Structures. J. Appl. Crystallogr. 1993, 26, 283-291.

(7) Laskowski, R. A.; Rullmannn, J. A.; MacArthur, M. W.; Kaptein, R.; Thornton, J. M. AQUA and PROCHECK-NMR: Programs for Checking the Quality of Protein Structures Solved by NMR. J. Biomol. NMR 1996, 8, 477-486. 